\title{
Detection of Structural Changes in Correctly Specified and Misspecified Conditional Quantile Polynomial Distributed Lag (QPDL) Model Using Change-point Analysis
}

\author{
Kwadwo Agyei Nyantakyi", B. L. Peiris, L. H. P. Gunaratne \\ Postgraduate Institute of Agriculture, University of Peradeniya, Sri Lanka
}

Copyright $@ 2015$ Horizon Research Publishing All rights reserved.

\begin{abstract}
Change-point analysis is a powerful tool for determining whether a change has taken place or not. In this paper we study the structural changes in the Conditional Quantile Polynomial Distributed Lag (QPDL) model using change-point analysis. We employ both the Binary Segmentation (BinSeg) and Cumulative Sum (Cusum) methods for this analysis. We studied the structural changes in both correctly specified and misspecified QPDL models. As an economic application we considered the production of rubber and its price returns. We observe that both Cusum and BinSeg methods correctly detected the structural changes for both the correctly specified and the misspecified QPDL model. The Cusum method gave the exact positions where the structural changes occurred and the BinSeg gave the approximated positions where the changes occurred. Both methods were able to detect the shift in time for both the mean and variance for the missspecified QPDL model, hence both methods were better for predicting structural stability in a QPDL models. The impact of this is that, when there are changes made to a data knowingly or unknowingly, they can be detected, as well as when these changes were effected. We further observed that both methods were powerful tools that better characterizes the changes, controls the overall error rate, robust to outliers, more flexible and simple to use.
\end{abstract}

Keywords Binseg, Cusum, Structural Changes, Misspecification, Mean-shift

\section{Introduction}

The question of structural stability of models is very important in predicting the future in various diverse areas of science such as economics, finance, physics, geology, medicine as well as in quality control and agriculture. Questions like did a change occur? Did more than one change occur? When did these changes occur? Can be answered by performing a change-point analysis, (Taylor, Wayne 2000a, 2000b)[13, 14].

In order to study the structural changes in models came the evolution of change-points which was introduced by the context of quality control by Csorgo and Horvath, (1997)[3].

Change-points has been employed in finding the possible changes in otherwise independent identically distributed random variables and has been extended to the stability tests of parameters of the regression functions, (Andrews, D. W. K. 1993)[1].

Killick, et al, (2012)[9], considered the common approach of detecting change-points through minimising a cost function over possible numbers and locations of change-points. They applied several established procedures for detecting changing points, such as penalised likelihood and minimum description length. In their study, they observed that Binary Segmentation is quicker as a search method, and believed this would be the case in almost all applications.

Auger and Lawrence (1989)[2] propose an alternative, exact search method for changepoint detection, namely the Segment Neighbourhood (SegNeigh) method which searches the entire segmentation space using dynamic programming. The problem with this exhaustive search method is that it has significant computational cost of $\mathrm{O}\left(Q_{n}{ }^{2}\right)$.

We use both the Binary Segmentation (BinSeg) and Cumulative Sum (cusum) methods for detecting the structural changes in the QPDL model because they are capable of detecting multiple changes (Mueller, 1992)[10]. The advantage of the BinSeg method is that it is computationally efficient, resulting in an $\mathrm{O}(\mathrm{n} \log \mathrm{n})$ calculation (Eckley et al, 2011)[4]. Both methods are powerful tools that better characterize the changes, control the overall error rate, robust to outliers, more flexible and simple to use, (Efron et al, 1993)[5]. 
The objectives of this study are, to detect the structural changes in correctly specified QPDL model, to detect the structural changes in misspecified QPDL model, and to study the mean-shift in time of the misspecified QPDL model. This study is important because as the part of our world moves into more sophisticated time periods, the accuracy of predicting the exact occurrences of future events as much as possible to reduce severe loses or impact is very important rather than using approximate estimates. It is also important in modeling to know when the exact change occurred and its impact.

\section{Materials and Methods}

\subsection{Data Source}

In order to answer the issues raised above, secondary annual data was collected from FAOSTAT, food balance sheet, price statistics, available with the Department of Census and Statistics Sri Lanka[6], and the World Bank (pink sheet)[15]. These data comprises of the production, imports, exports and prices of rubber. The rubber data ranges from 1961-2011.

\subsection{Statistical Software}

The $\mathrm{R}$ software, with the package 'Change-point' was used in analysing the structural changes in the conditional quantile polynomial distributed lag models.

\subsection{Methodology}

Testing for structural changes

We assume two (2) conditional $2^{\text {nd }}$ degree polynomial $\alpha$-quantile regimes

$$
\begin{aligned}
Y_{1 t}=\varphi(\alpha) & +a_{1,0}(\alpha) Z_{1,0 t}+a_{1,1}(\alpha) Z_{1,1 t}+a_{1,2}(\alpha) Z_{1,2 t} \\
& +\varepsilon_{1 t}, \quad t=1,2, \ldots, T_{1} \\
Y_{2 t}= & \varphi(\alpha)+a_{2,0}(\alpha) Z_{2,0 t}+a_{2,1}(\alpha) Z_{2,1 t} \\
& +a_{2,2}(\alpha) Z_{2,2 t},+\varepsilon_{2 t}, \quad t=1,2, \ldots, T_{2}
\end{aligned}
$$

With $\left\{\varepsilon_{1 t}\right\}$ and $\left\{\varepsilon_{1 t}\right\}$ i.i.d unobservable innovations with

$$
\begin{aligned}
E\left(\varepsilon_{1 t}\right)=0 \text { and } E\left(\varepsilon_{2 t}\right) & =0, V\left(\varepsilon_{1 t}\right) \\
= & \sigma_{1}^{2} \text { and } V\left(\varepsilon_{2 t}\right)=\sigma_{2}^{2}
\end{aligned}
$$

We further assume that $E\left(\varepsilon_{1 t}\right)$ and $E\left(\varepsilon_{2 t}\right)$ are i.i.d innovations which are independent of each other.

Let $\hat{a}_{1,0}(\alpha)$ and $\hat{a}_{2,0}(\alpha)$ be the estimates of $a_{1,0}(\alpha)$ and $a_{2,0}(\alpha)$ respectively. Then we also define

$$
Y_{1 t}^{*}=Y_{1 t}-\bar{Y}_{1} \text { and } Y_{2 t}^{*}=Y_{2 t}-\bar{Y}_{1}
$$

Then the hypothesis can be tested as follows:

\section{Hypothesis:}

$$
\begin{aligned}
& H_{0}: a_{1,0}(\alpha)=a_{2,0}(\alpha) \text { and } H_{1}: a_{1,0}(\alpha) \neq a_{2,0}(\alpha) \\
& \text { (Assuming } \sigma_{1}^{2}=\sigma_{2}^{2} \text { ) } \\
& \text { Then under } H_{0} \text { we have, }
\end{aligned}
$$

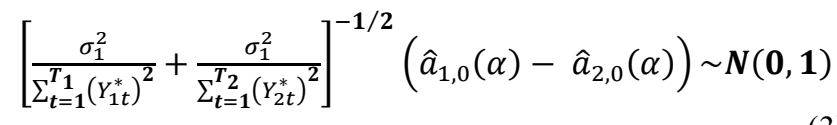

Let $\left(\hat{\varepsilon}_{1 t}\right)$ and $\left(\hat{\varepsilon}_{2 t}\right)$ be the residuals estimated respectively, then $\frac{\Sigma_{t=1}^{T_{1}} \hat{\varepsilon}_{1 t}^{2}}{\sigma_{1}^{2}} \sim \chi_{t-2}^{2}$ and $\frac{\Sigma_{t=1}^{T_{2}} \hat{\varepsilon}_{2 t}^{2}}{\sigma_{2}^{2}} \sim \chi_{t-2}^{2}$

and therefore we have

$$
\frac{\sum_{t=1}^{T_{1}} \hat{\varepsilon}_{1 t}^{2}}{\sigma_{1}^{2}}+\frac{\sum_{t=1}^{T_{2}} \hat{\varepsilon}_{2 t}^{2}}{\sigma_{2}^{2}} \sim \chi_{t-4}^{2}
$$

Where we have $\boldsymbol{T}_{1}+\boldsymbol{T}_{2}=\boldsymbol{T}$ and since (3) and (4) are independent we have

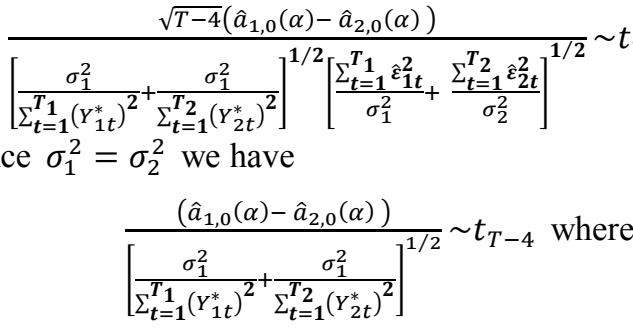

$$
\begin{aligned}
& \hat{\sigma}^{2}=(T-4)^{2}\left(\sum_{t=1}^{T_{1}} \hat{\varepsilon}_{1 t}^{2}+\sum_{t=1}^{T_{2}} \hat{\varepsilon}_{2 t}^{2}\right)
\end{aligned}
$$

Hence $H_{0}$ can be tested using either one tail or two tail depending on the $H_{1}$.

Now we apply the change point algorithm to see when and where the changes in the QPDL model occurred, using cumulative sum (cusum) and Binary Segmentation (BinSeg) methods.

\section{Description of the test procedure for the detection of the change-point}

Let us now consider a conditional $2^{\text {nd }}$ degree polynomial $\alpha$-quantile model with a change after an unknown time point

$$
\begin{gathered}
1 \leq k^{*}=k^{*}(n) \leq n \\
X_{i t}= \begin{cases}Z_{i t} & t \leq k^{*} \\
Y_{i t-k^{*}} & t>k^{*}\end{cases}
\end{gathered}
$$

Where $\left\{Y_{i t}\right\}$ is some QPDL which differs distributionally from $\left\{Z_{i t}\right\}$. The unknown parameter $k^{*}$ is called the change-point.

We are now interested in the testing problem

$$
H_{0}: k^{*}=n \text { vs. } H_{1}: k^{*}<n
$$

Our testing procedures are based on various functionals of the partial sums of estimated

residuals with respect to the model (1)

$$
\begin{gathered}
\hat{S} n(k)=\sum_{t=p+1}^{k} \hat{\varepsilon}_{t} \\
=\sum_{t=p+1}^{k}\left(X_{i t}-f\left(Z_{i t}, \hat{\beta}_{n}\right)\right) .
\end{gathered}
$$

where $\hat{\beta}_{n}$ is the least-squares estimator of $\beta_{0}$ (assuming the null hypothesis holds true).

Precisely we minimize the nonlinear least squares (NLLS) with respect to $\beta$. 


$$
\begin{gathered}
Q_{n}(\beta)=\sum_{t=p+1}^{k}\left(X_{t}-f\left(Z_{i t}, \hat{\beta}_{n}\right)\right)^{2} \\
=\sum_{t=p+1}^{k} q_{n}(\beta)
\end{gathered}
$$

Thus we consider the nonlinear least squares estimator

$$
\hat{\beta}_{n}=\arg \min _{\beta \epsilon k} Q_{n}(\beta)
$$

for a suitable compact set $\mathrm{K}$. The minimization is usually obtained by solving the nonlinear score function

$$
\frac{\partial Q_{n}\left(\widehat{\beta}_{n}\right)}{\partial \beta}=0 \text {. }
$$

Which yields

$$
\sum_{t=p+1}^{k} \hat{\varepsilon}_{t}=0 .
$$

The behaviour of the estimator $\beta_{n}$ is investigated in a variety of situations including the correctly specified case without change as well as possibly misspecified cases with and without change. According to Horvath et al (2004)[8] and $\mathrm{Wu},(2004)[16]$, under appropriate assumptions $\hat{\beta}_{n}$ is eventually in the interior of the compact set $\mathrm{K}$, so that we can assume for limit considerations.

In fact, if $\hat{\beta}_{n}$ is not in the interior of K, (Hinkley, D. V. 1971)[7], we will reject the null hypothesis immediately since either a change occurred or the model is not capable of modeling the observed time series sufficiently well.

Test statistics are of the form

$$
\begin{aligned}
& T_{n 1}=\max _{p<k<n}\left(\sqrt{\left(\frac{n-p}{k(n-p-k)}\right)}\left|\hat{S}_{n}(k)\right|\right), \\
& T_{n 2}(q)=\max _{p<k<n}\left(\frac{1}{\sqrt{n-p} q\left(\frac{k}{n-p}\right)}\left|\hat{S}_{n}(k)\right|\right),
\end{aligned}
$$

$$
\begin{gathered}
T_{n 3}(G)=\max _{p+G<k<n} \frac{1}{\sqrt{G}}\left(\left|\hat{S}_{n}(k)-\hat{S}_{n}(k-G)\right|\right), \\
T_{n 1}=\max _{p+G<k<n-p-G} \frac{1}{\sqrt{2 G}}\left(\mid \hat{S}_{n}(k+G)-2 \hat{S}_{n}(k)+\right. \\
\left.\hat{S}_{n}(k-G) \mid\right), \\
T_{n 4}(r)=\frac{1}{n-p} \sum_{k=p+1}^{n-1} \frac{1}{r(k /(n-p))}\left(\frac{1}{\sqrt{n-p}} \hat{S}_{n}(k)\right)^{2},
\end{gathered}
$$

where $\mathrm{q}(\cdot)$ and $\mathrm{r}(\cdot)$ are weight functions defined on $(0,1)$ specified below and $\mathrm{G}<\mathrm{n}$. In this case, we obtain a consistent change-point estimator which is related to the test statistics. (Stockis, J.-P et al, 2010, Tadjuidje K., J. et al, 2011)[11, 12]

\section{Results and Discussions}

\begin{tabular}{|c|c|c|c|c|c|c|c|c|}
\hline \multirow{2}{*}{$\frac{\text { tau }}{0.25}$} & \multirow{2}{*}{$\begin{array}{c}\text { Change type } \\
\text { Cusum }\end{array}$} & \multicolumn{6}{|c|}{ Positions of the change } & \multirow{2}{*}{$\frac{\text { Max no. of change }}{5}$} \\
\hline & & 16.00 & 41.00 & 44.00 & 46.00 & 24.00 & & \\
\hline & BinSeg & 16 & 19 & 23 & 42 & 44 & 48 & 5 \\
\hline & Mean & 1572.5 & 1000.9 & 1539.4 & 1028.4 & 1432.9 & 1829.5 & \\
\hline & Variance & 52268.88 & 42726.38 & 36481.89 & 22493.61 & 570.39 & 30727.15 & \\
\hline
\end{tabular}

\section{Detection of structural Changes for the correctly specified QPDL model}

Detection of structural changes in the QPDL model before misspecification are shown on tables $1-4$, using the methods Cumulative Sum (cusum) and Binary Segmentation (BinSeg) with Schwarz Information Criterion (SIC) penalty.

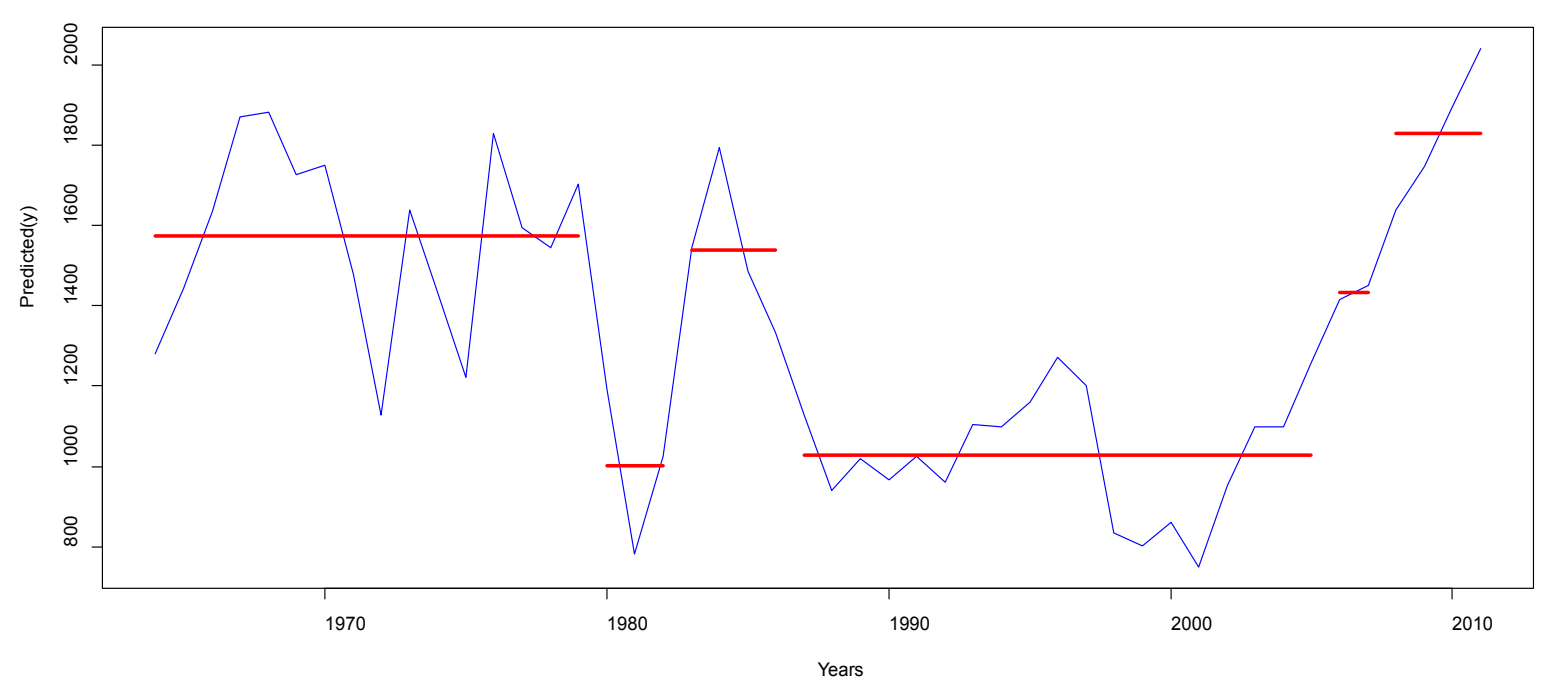

Figure 1. a plot showing the changes in production of Predicted $\hat{y}$ against Years of tau $=0.25$

Table 1. Conditional quantile polynomial distributed lag (QPDL), tau $=0.25$ showing the maximum number of changes, position of the change in both the mean and variance for cusum and BinSeg methods. (SIC (Penalty) $=7.742402$ ) 
From table 1, we observe that, both the cumulative sum (Cusum) and the Binary Segmentation (BinSeg) methods detected maximum of 5 structural changes. The Cusum gives the exact change at various positions whereas the BinSeg gives the approximated positions. The minimum change for both methods is at the $16^{\text {th }}$ position and the highest maximum for Cusum is $46^{\text {th }}$ and that of BinSeg is $48^{\text {th }}$ position. There changes in mean production with minimum of 1000.9, occurring at $19^{\text {th }}$ position and the maximum mean of 1829.5 with a change at $48^{\text {th }}$ position. The variance changes associated with the changes are shown in the last row with minimum variance of 570.4 and for a mean of 1432.9 at the $44^{\text {th }}$ position and a maximum variance of 52268.9 for a mean of 1572.5 at the $16^{\text {th }}$ position. figure 1 showing the various positions and the time period for which each change occurred.

From figure 1, we observe the $1^{\text {st }}$ change occurred in 1979 , showing a high drop in average production to a record low. The $2^{\text {nd }}$ change in production occurred in 1982 with a high increase in average production again. The $3^{\text {rd }}$ change shows another drop in average production which lasted from 1977 to 2005 and then the $4^{\text {th }}$ change in average production occurred. There was an increase in average production from 2005 to 2008 and then a change occurred, and then a subsequent increase till 2011.

Table 2, we observed that, both methods detected 5 optimum changes, with the $1^{\text {st }}$ change occurring at the $16^{\text {th }}$ position for both methods. The minimum average change in production 1013.1 occurred at $19^{\text {th }}$ position with a variance of 148533.5 and the highest average mean production 2579 occurred at $48^{\text {th }}$ position with variance of 42430 . The graphical interpretation of the change is shown on figure 2.

Similarly, we observe from figure 2 , the $1^{\text {st }}$ change occurred in 1979, showing a high drop in average production to a record low. The $2^{\text {nd }}$ change in production occurred in 1982 with a high increase in average production again. The $3^{\text {rd }}$ change shows another drop in average production which lasted from 1977 to 2005 and then the $4^{\text {th }}$ change in average production occurred. There was an increase in average production from 2005 to 2008 and then a change occurred, and then a subsequent increase till 2011.

Table 2. Conditional quantile polynomial distributed lag (QPDL) tau $=0.50$ showing the maximum number of changes, position of the change in both the mean and variance for cusum and BinSeg methods. (SIC (Penalty) =7.742402)

\begin{tabular}{|c|c|c|c|c|c|c|c|c|}
\hline tau & Change type & \multicolumn{6}{|c|}{ Positions of the change } & Max no. of change \\
\hline 0.50 & Cusum & 16.00 & 41.00 & 45.00 & 47.00 & 3.00 & & 5 \\
\hline & BinSeg & 16 & 19 & 23 & 42 & 45 & 48 & 5 \\
\hline & Mean & 2001.05 & 1013.08 & 1953.29 & 1177.79 & 1998.99 & 2579.01 & \\
\hline & Variance & 137322.4 & 148533.48 & 105870.46 & 65529.7 & 27957.45 & 42430.54 & \\
\hline
\end{tabular}

Changepoint at tau(0.50)

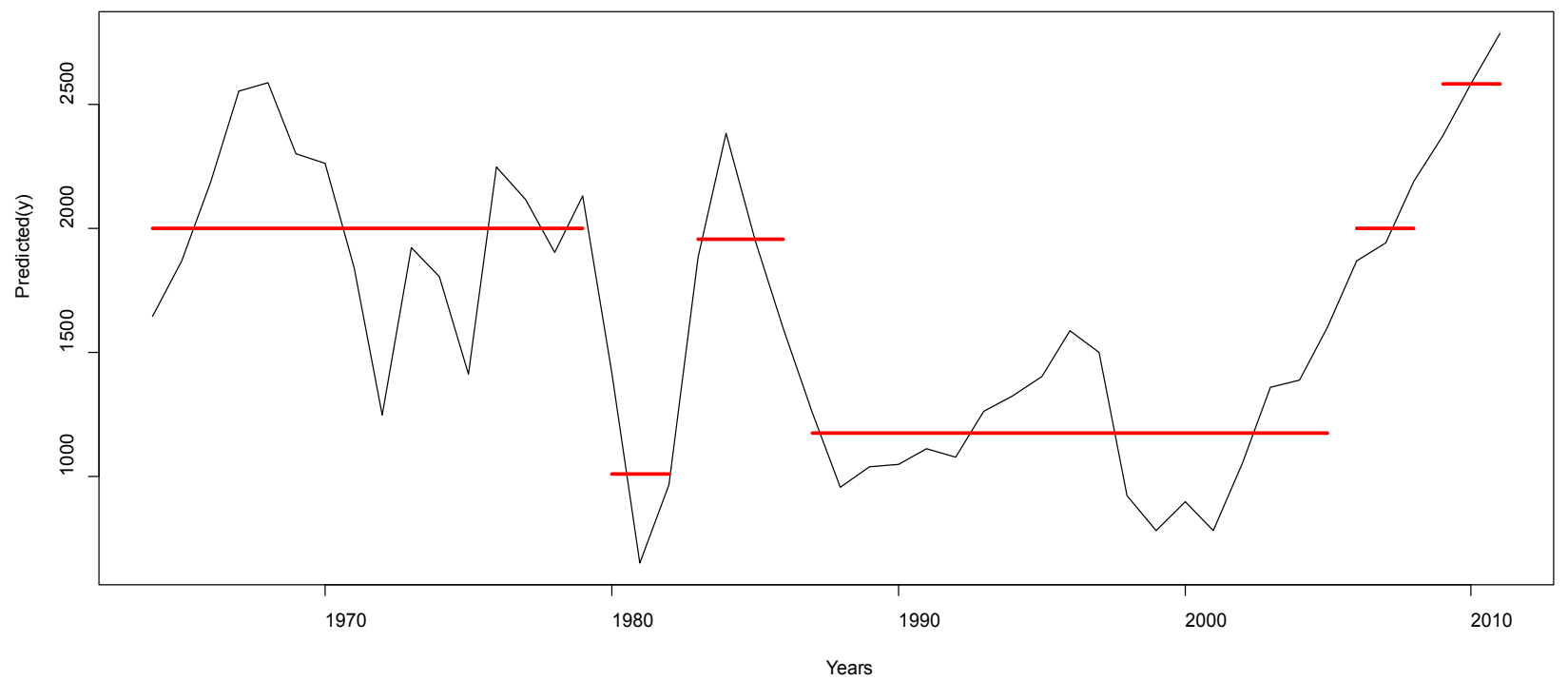

Figure 2 a plot showing the changes in production of Predicted $\hat{y}$ against Years of tau $=0.50$

Table 3. Conditional quantile polynomial distributed lag (QPDL) tau $=0.75$ showing the maximum number of changes, position of the change in both the mean and variance for cusum and BinSeg methods. (SIC (Penalty) $=7.742402$ )

\begin{tabular}{|c|c|c|c|c|c|c|c|}
\hline tau & Change type & \multicolumn{5}{|c|}{ Positions of the change } & Max no. of change \\
\hline 0.75 & Cusum & 17.00 & 42.00 & 45.00 & 24.00 & 19.00 & 5 \\
\hline & BinSeg & 17 & 23 & 42 & 44 & 48 & 4 \\
\hline & Mean & 2488.207 & 1987.198 & 1362.633 & 2166.128 & 2965.220 & \\
\hline & Variance & 164495.50 & 533186.09 & 86739.77 & 18553.91 & 146672.70 & \\
\hline
\end{tabular}




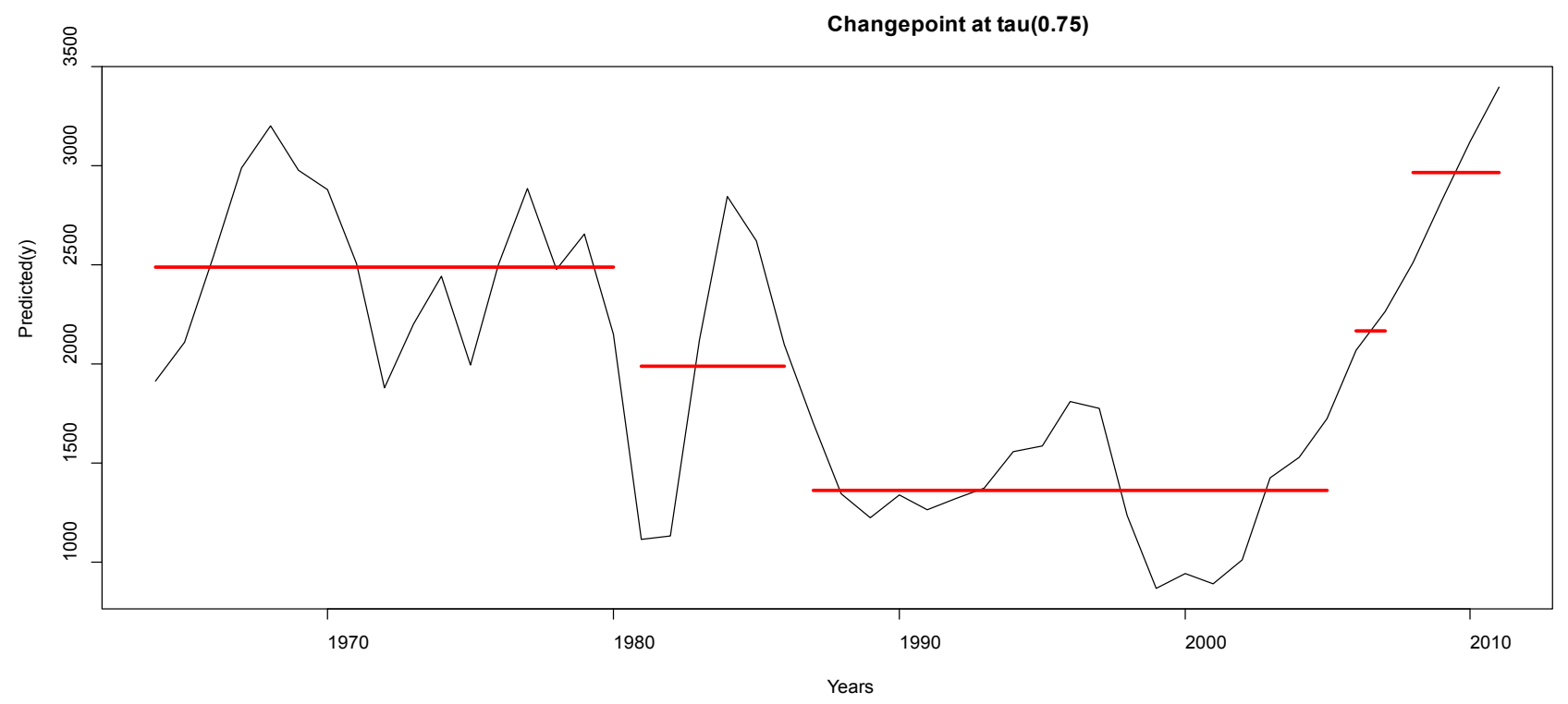

Figure 3. a plot showing the changes in production of Predicted $\hat{y}$ against Years of tau=0.75

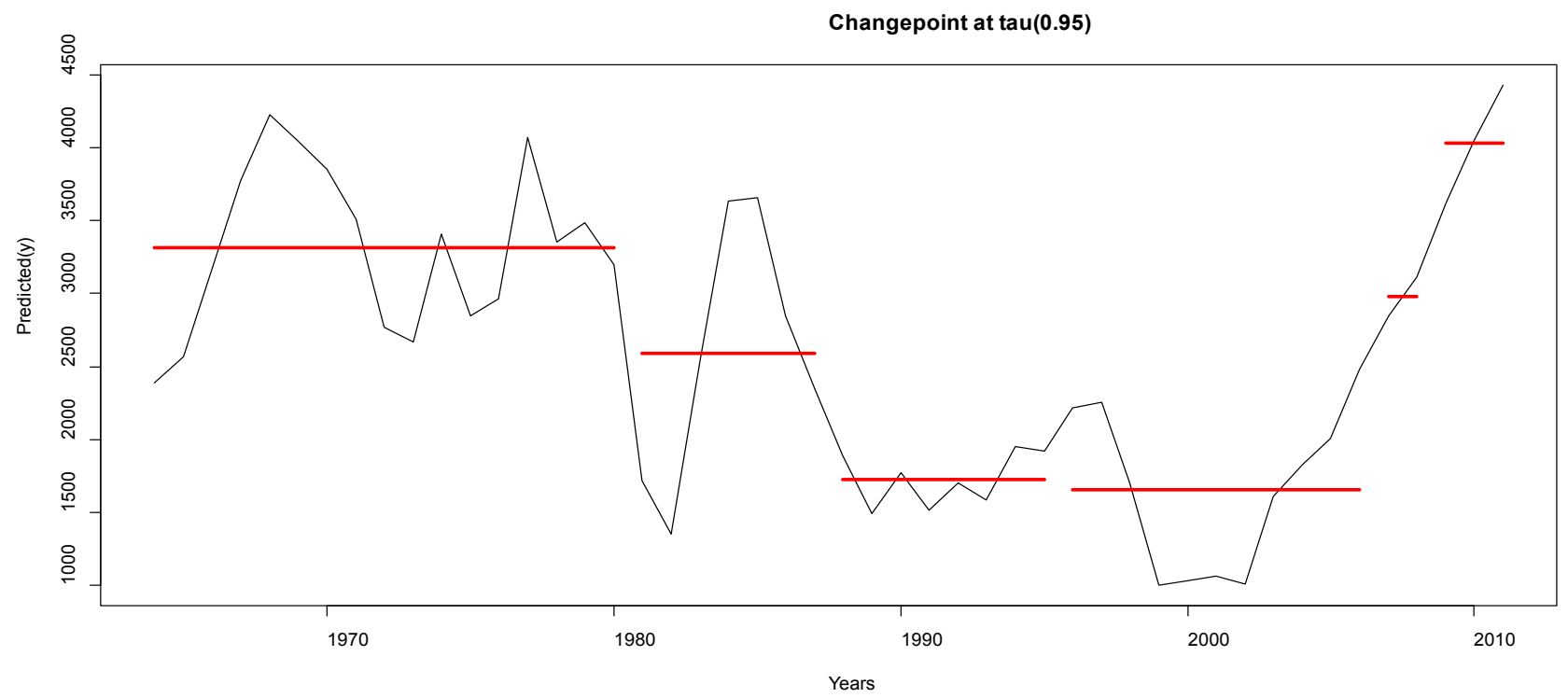

Figure 4. a plot showing the changes in production of Predicted $\hat{y}$ against Years of tau $=0.95$

Table 4. Conditional quantile polynomial distributed lag $(\mathrm{QPDL})$ tau $=0.95$ showing the maximum number of changes, position of the change in both the mean and variance for cusum and BinSeg methods. (SIC (Penalty) $=7.742402$ )

\begin{tabular}{|c|c|c|c|c|c|c|c|c|}
\hline tau & Change type & \multicolumn{9}{|c|}{ Positions of the change } & Max no. of change \\
\hline 0.95 & Cusum & 23.000 & 42.000 & 45.000 & 46.000 & 17.000 & & 5 \\
\hline & BinSeg & 17 & 24 & 32 & 43 & 45 & 48 & 5 \\
\hline & Mean & 3311.961 & 2586.547 & 1728.211 & 1654.949 & 2978.641 & 4032.445 & \\
\hline & Variance & 313650.7 & 773252.0 & 33320.35 & 309450.68 & 33541.0 & 164135.34 & \\
\hline
\end{tabular}

Table 3 shows an optimum of 5 changes for Cusum and 4 for BinSeg. The minimum average production 1362.6 occurred at the $42^{\text {nd }}$ position with a variance of 86739.8 and the maximum average production 2965.2 occurring at the $48^{\text {th }}$ position with variance of 146672.7 . The graphical interpretation is shown by figure 3 .

We observe from figure 3 , the $1^{\text {st }}$ change occurred in 1981, with a drop in average production between 1981 and 1986. The $2^{\text {nd }}$ change in average production occurred in 1986 and 2005 with a high decrease in average production again. The $3^{\text {rd }}$ change shows another an average increase in production which lasted from 2005 to 2007 and then the $4^{\text {th }}$ change in average production occurred. There was an increase in average production from 2005 till 2011.

Table 4, we observed that, both methods detected 5 optimum changes, with the $1^{\text {st }}$ change occurring at the $23^{\text {rd }}$ position for Cusum and $17^{\text {th }}$ position for BnSeg. The minimum average change in production was 1655.1 and occurred at $43^{\text {rd }}$ position with a variance of 309451 and the highest average mean production was 4032.4 and occurred at 
$48^{\text {th }}$ position with variance of 164135 . The graphical interpretation of the change is shown on figure 4.

Similarly, we observe from figure 2 , the $1^{\text {st }}$ change occurred in 1980, showing a drop in average production between 1980 and 1988. The $2^{\text {nd }}$ change in production occurred in 1988 with a further decrease in average production. The $3^{\text {rd }}$ change shows another slight drop in average production which lasted from 1997 to 2006 and then the $4^{\text {th }}$ change in average production occurred. There was an increase in average production from 2006 to 2008 and then a change occurred, and then a subsequent increase till 2011.

\section{Detection of structural Changes for the misspecified QPDL model}

Detection of structural changes in the misspecified QPDL model are shown in tables 5-8, using the methods Cumulative Sum (cusum) and Binary Segmentation (BinSeg) with Schwarz Information Criterion (SIC) penalty.

From table 5, we observe that, both the cumulative sum
(Cusum) and the Binary Segmentation (BinSeg) methods detected maximum of 5 structural changes. Here there is a shift in position of the $1^{\text {st }}$ change from the $16^{\text {th }}$ position from the correctly specified model to $23^{\text {rd }}$ position for the misspecified model for both methods. The minimum change in mean production was 828.48 , occurring at $39^{\text {th }}$ position with variance of 2161 . The maximum average production of 1494.7 with a variance of 51195.8 occurred at $23^{\text {rd }}$ position. Below is figure 5 showing the various positions and the time period for which each change occurred.

Similarly, from figure 5, we observe a shift of the change in the average production which occurred in 1979 for the correctly specified QPDL model to 1988 in the misspecified QPDL model. There was high drop in the mean production between 1988 and 1996 and then an increase occurred for a short period in 1997. There was a drop again between 1998 and 2003. There was an increase in average production in 2004. There was a further high increase in production from 2005 till 2011.

Table 5. Misspecified Conditional quantile polynomial distributed lag (QPDL), tau=0.25 showing the maximum number of changes, position of the change in both the mean and variance for cusum and BinSeg methods. SIC (Penalty) $=7.700295$

\begin{tabular}{|c|c|c|c|c|c|c|c|c|}
\hline tau & Change type & \multicolumn{6}{|c|}{ Positions of the change } & Max no. of change \\
\hline 0.25 & Cusum & 23.000 & 41.000 & 45.000 & 43.000 & 15.000 & & 5 \\
\hline & BinSeg & 23 & 31 & 33 & 39 & 41 & 47 & 5 \\
\hline & Mean & 1494.72 & 974.70 & 1109.92 & 828.48 & 956.63 & 1463.46 & \\
\hline & Variance & 51195.76 & 7094.85 & 233.45 & 2160.53 & 905.68 & 49666.63 & \\
\hline
\end{tabular}

Changepoint at ktau(0.25)

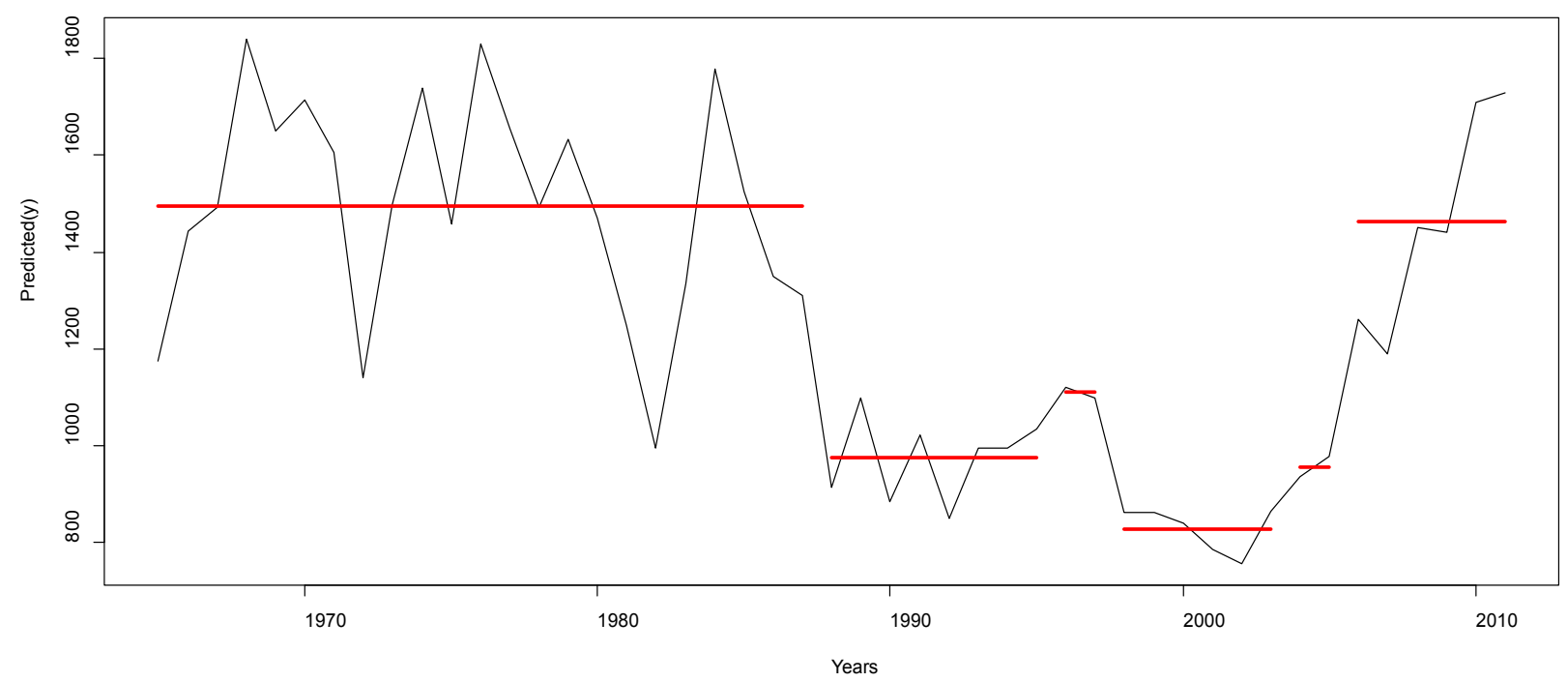

Figure 5. a plot showing the changes in production of Predicted $\hat{y}$ against Years of tau=0.25

Table 6. Conditional quantile polynomial distributed lag (QPDL) misspecified tau $=0.50$ showing the maximum number of changes, position of the change in both the mean and variance for cusum and BinSeg methods. SIC (Penalty) $=7.700295$

\begin{tabular}{|c|c|c|c|c|c|c|c|}
\hline tau & Change type & \multicolumn{5}{|c|}{ Positions of the change } & Max no. of change \\
\hline 0.50 & Cusum & 21.000 & 40.000 & 43.000 & 41.000 & 42.000 & 5 \\
\hline & BinSeg & 21 & 41 & 47 & & & 3 \\
\hline & Mean & 1766.641 & 1311.461 & 1877.811 & & & \\
\hline & Variance & 111066.87 & 30018.25 & 34896.11 & & & \\
\end{tabular}


Similarly, from table 6, we observe that, for the misspecified QPDL model, the cumulative sum (Cusum) detected a maximum of 5 structural changes and the Binary Segmentation (BinSeg) methods detected only 3 structural changes at the $50^{\text {th }}$ percentile. Whereas in the correctly specified model, both methods detected 5 structural changes at the $50^{\text {th }}$ percentile. Here there is a shift in position of the $1^{\text {st }}$ change from the $16^{\text {th }}$ position from the correctly specified model to $21^{\text {st }}$ position for the misspecified model for both methods. The change in minimum average production was 1311.461, occurring at $41^{\text {st }}$ position with variance of 30018.25. The maximum average production of 1877.811 with a variance of 34896.11 occurred at $47^{\text {rd }}$ position. Below is figure 6 , showing the various positions and the time period for which each change occurred.

Similarly, from figure 6 , we observe a shift of the change in the average production which occurred in 1979 for the correctly specified QPDL model to 1987 in the misspecified QPDL model. There was high drop in the mean production between 1988 and 2005 and then followed by a high increase in average production occurred from 2005 till 2011.
From table 7, we observe that, both the cumulative sum (Cusum) and the Binary Segmentation (BinSeg) methods detected maximum of 5 structural changes. Similarly, there is a shift in position of the $1^{\text {st }}$ change from the $16^{\text {th }}$ position from the correctly specified model to $21^{\text {st }}$ position for Cusum and $15^{\text {th }}$ position for BinSeg for the misspecified model. The change in minimum mean production was 1471.1, occurring at $41^{\text {st }}$ position with variance of 56549 . The maximum average production of 2594.9 with a variance of 44469.3 occurred at $47^{\text {th }}$ position. Below is figure 7 showing the various positions and the time period for which each change occurred.

Similarly, from figure 7, we observe a shift of the change in the average production which occurred in 1981 for the correctly specified QPDL model to 1979 in the misspecified QPDL model which lasted till 1985 and then there was a further drop in average production 1987. There was high drop in the mean production between 1988 and 2005, and then an increase occurred for a short period in 2006. There was a further high increase in production from 2007 till 2011.

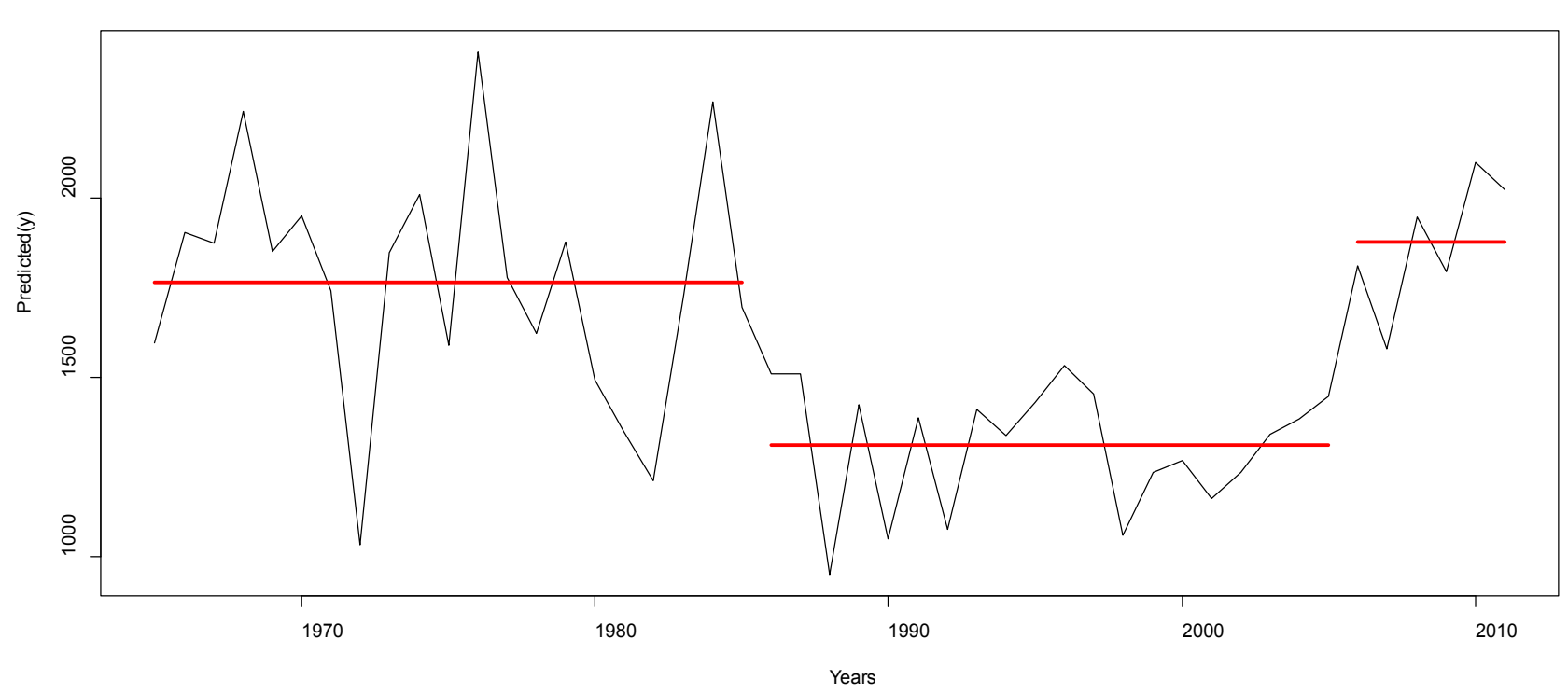

Figure 6. a plot showing the changes in production of Predicted $\hat{y}$ against Years of tau=0.50

Table 7. Conditional quantile polynomial distributed lag (QPDL) misspecified tau $=0.75$ showing the maximum number of changes, position of the change in both the mean and variance for cusum and BinSeg methods. SIC (Penalty) $=7.700295$

\begin{tabular}{|c|c|c|c|c|c|c|c|c|}
\hline tau & Change type & \multicolumn{9}{|c|}{ Positions of the change } & Max no. of change \\
\hline 0.75 & Cusum & 21.000 & 41.000 & 45.000 & 43.000 & 42.000 & 5 \\
\hline & BinSeg & 15 & 21 & 23 & 41 & 43 & 47 & 5 \\
\hline & Mean & 2372.246 & 2048.590 & 1891.935 & 1471.076 & 2141.091 & 2594.873 & \\
\hline & Variance & 200317.63 & 364103.87 & 189.03 & 56549.01 & 40653.84 & 44469.31 & \\
\hline
\end{tabular}




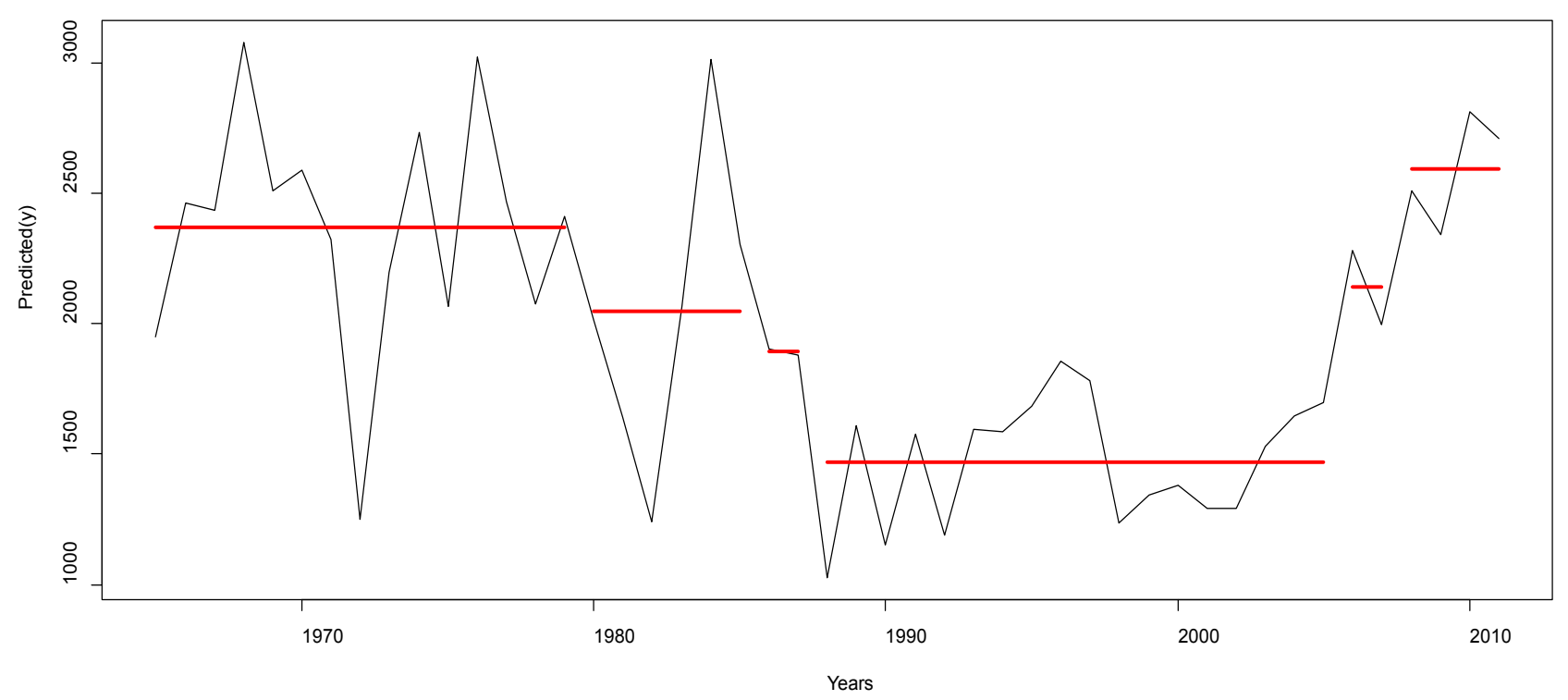

Figure 7. a plot showing the changes in production of Predicted $\hat{y}$ against Years of tau $=0.75$

Table 8. Conditional quantile polynomial distributed lag (QPDL) misspecified tau $=0.95$ showing the maximum number of changes, position of the change in both the mean and variance for cusum and BinSeg methods. SIC (Penalty) $=7.700295$

\begin{tabular}{|c|c|c|c|c|c|c|c|c|}
\hline tau & Change type & \multicolumn{9}{|c|}{ Positions of the change } & Max no. of change \\
\hline 0.95 & Cusum & 23.000 & 41.000 & 45.000 & 43.000 & 16.000 & & 5 \\
\hline & BinSeg & 16 & 21 & 23 & 41 & 43 & 47 & 5 \\
\hline & Mean & 3774.566 & 3094.037 & 2968.266 & 1692.618 & 2822.483 & 3979.721 & \\
\hline & Variance & 511265.64 & 1443470.48 & 7900.62 & 142103.32 & 24939.93 & 273117.81 & \\
\hline
\end{tabular}

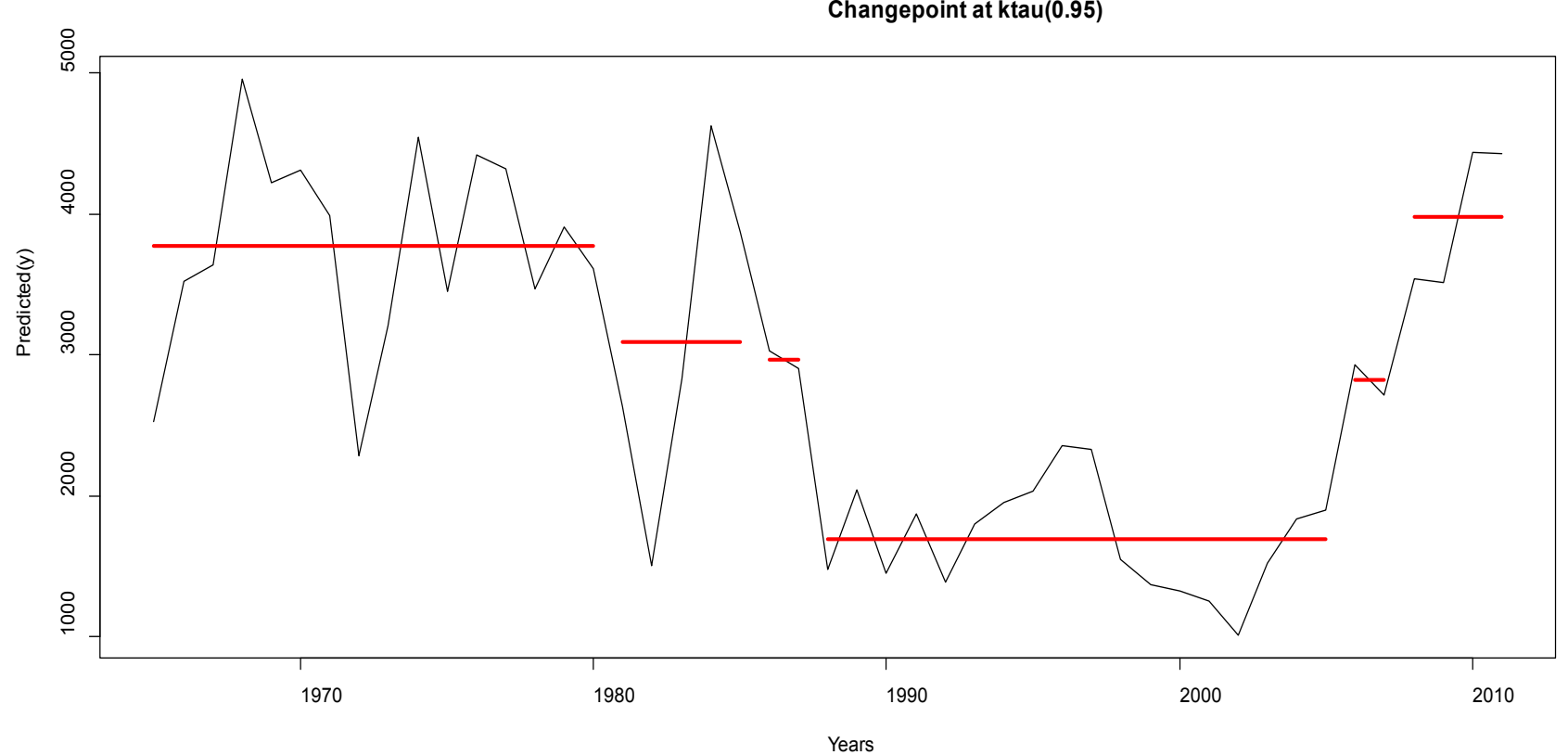

Figure 8. a plot showing the changes in production of Predicted $\hat{y}$ against Years of tau $=0.95$

From table 8, we observe that, both the cumulative sum (Cusum) and the Binary Segmentation (BinSeg) methods detected maximum of 5 structural changes. Similarly, there is a shift in position of the $1^{\text {st }}$ change from the $23^{\text {rd }}$ position for Cusum and $17^{\text {th }}$ position for BinSeg from the correctly specified model to $23^{\text {rd }}$ position for Cusum and $16^{\text {th }}$ position for BinSeg for the misspecified model. The change in minimum mean production was 1692.6 , occurring at $41^{\text {st }}$ position with variance of 142103.3 . The maximum average production of 3979.7 with a variance of 273117.8 occurred at $47^{\text {th }}$ position. Below is figure 8 showing the various positions and the time period for which each change 
occurred.

Similarly, from figure 8 , we observe a shift of the change in the average production which occurred in 1980 for the correctly specified QPDL model to 1979 in the misspecified QPDL model. There was a drop in the mean production between 1979 and 1986 and then a decrease occurred for a short period in 1987. There was a high drop again between 1988 and 2005. There was an increase in average production in 2006. There was a further high increase in production from 2007 till 2011.

\section{Conclusions}

From the analysis, we observed that both methods detected the exact time change and the magnitude of the change. We also observed that both the Cusum and the BinSeg methods detected the structural changes for both the correctly specified and the misspecified QPDL model. The Cusum method gives the exact positions where the structural changes occurred and the BinSeg gives the approximated positions where the changes occurred. Both methods were able to detect the shift in time for both the mean and variance for the missspecified QPDL model, hence both methods were better for predicting structural stability in a QPDL models. The impact of this is that, when there are changes made to a data knowingly or unknowingly, they can be detected, as well as when these changes were effected. We further observed that both methods were powerful tools that better characterizes the changes, controls the overall error rate, robust to outliers, more flexible and simple to use.

\section{REFERENCES}

[1] Andrews, D. W. K. (1993) Test for parametric instability and structural change with unknown change point. Journal of theoretical probability, 61:821-856.

[2] Auger, I. E. And Lawrence, C. E. (1989) Algorithms for the Optimal Identification of Segment Neighborhoods, Bulletin of Mathematical Biology 51(1), 39-54
[3] Csorgo, M., Horvath., L. (1997) Limit Theorems in Change-Point Analysis, Wiley E. S. Page (1954) Continuous Inspection Schemes, Biometrika 41(1/2), 100-115

[4] Eckley, I. A., Fearnhead, P., and Killick, R. (2011). Analysis of changepoint models. In Barber, D., Cemgil, T., and Chiappa, S., editors, Bayesian Time Series Models. Cambridge University Press.

[5] Efron, Bradley and Tibshirani, Robert (1993), An introduction to the Bootstrap, Chapman \& Hall, New York.

[6] FAOSTAT, Sri Lanka Annual Data(1961-2011),[on line].[Accessed on 10.02.2014]. Available at http://faostat.fao.org

[7] Hinkley, D. V. (1971), "Inference about the change-point from cumulative sum tests," Biometrika, 58 3, 509-523.

[8] Horvath, L., Huskova, M., Kokoszka, P., and Steinebach, J.( 2004) Monitoring changes in linear models. J. Statist. Plann. Inference, 126:225 - 251.

[9] Killick, R., Fearnhead, P., Eckley, I. A., (2012), "Optimal detection of changepoints with a linear computational cost", Journal of the American Statistical Association 107 (500), $1590-1598$

[10] Mueller, H.-G. (1992) Change points in nonparametric regression analysis. Annals of Statistics, 20:737-761.

[11] Stockis, J.-P., Franke, J., Tadjuidje K. J., (2010) "On geometric ergodicity of charme models". Journal of Time Series Analysis, 31:141-152.

[12] Tadjuidje K., J Kirch, C.(2011) Testing for parameter stability in nonlinear autoregressive models. Preprint,

[13] Taylor, Wayne (2000a), Change-Point Analyzer 2.0 shareware program, Taylor Enterprises, Libertyville, Illinois. Web: http://www.variation.com/cpa

[14] Taylor, Wayne (2000b), “A Pattern Test for Distinguishing Between Autoregressive and Mean-Shift Data," submitted to Journal of Quality Technologies.

[15] World Bank Pink Sheet Annual Data (1961-2013), [on line]. [Accessed on 08.08.2014]. Available at http://econ.worldbank.org

[16] Wu, Y. (2004), Inference for change point and post change mean after a CUSUM test. Springer, New York. 